\title{
Der neue Gesetzentwurf über ärztliche Ehrengerichte.
}

Wir haben den neuen "Entwurf eines Gesetzes, betreffend die ärztlichen Ehrengerichte, das Umlagerecht und die Kassen der Aerztekammern" im Anzeigentheile der vorigen Nummer seinem vollen Wortlaute nach veröffentlicht. Unsere Leser sind dadurch in den Stand gesetzt, den Text des neuen Entwurfes mit dem ursprünglichen, in einer Sonderbeilage von No. 13 des vorigen Jahrganges bekannt gemachten zu vergleichen und sich ein Urtheil darüber zu bilden, wie weit die Regierung den von den berufenen Vertretern der preussischen Aerzteschaft formulirten Wünschen und Vorschlägen in den entscheidenden Punkten nach-, oder wenigstens entgegengekommen ist.

Bekanntlich waren es der Hauptsache nach vier Punkte, die nicht bloss bei prinzipiellen Gegnern, sondern auch bei vielen Anhängern und Freunden der Ehrengerichte Anstoss erregten und der allseitigen Verständigung namhafte Schwierigkeiten zu bereiten drohten. Der erste Punkt betraf die in $\S 2$ des ersten Abschnittes ausgesprochene Exemtion der beamteten Aerzte und Sanitätsoffiziere (sowie der Saniitätsoffiziere des Beurlaubtenstandes während ihrer Einziehung zur Dienstleistung). Man nahm mit vollem Rechte besonders Anstoss daran, dass die beamteten Aerzte zwar selbst der collegialen Gerichtsbarkeit nicht unterstehen, dagegen von dem Rechte der Ausübung dieser Gerichtsbarkeit keineswegs ausgeschlossen sein sollten. Dieser auch als Gefahr empfundenen Ungleichheit wird nun durch den neuen Zusatz zu $\S 2$ die Spitze abgebrochen, die lautet: "Die nach Absatz 1 der Zuständigkeit des Ehrengerichtes nicht unterworfenen Aerzte sind bei den Wahlen für das Ehrengericht weder wahlberechtigt noch wählbar."

Man hat sich mit diesem durch die Gerechtigkeit geforderten Zugeständnisse zwar einverstanden erklärt, alsbald aber den Einwand erhoben, die von den Theilnehmern der Ehrengerichte ausgeschlossenen beamteten Aerzte u. s. w. würden trotzdem a uf Grund königlicher Ernennung (nach $\S 43$ ) Mitglieder des Ehrengerichth ofes werden konnen, somit in die Lage kommen, bei Entscheidungen über ihre nicht eximirten Collegen in der $\mathrm{Be}$ rufungsinstanz mitzuwirken. Ich muss gestehen, dass mir eine derartige Annahme als vollig undenkbar, mit dem gesunden Menschenverstande, sowie mit dem Geiste und Wortlaute des Entwurfes schlechterdings unvereinbar erscheint. Es kann unmöglich in der Absicht der Regierung liegen, Aerzten, die den Ehrengerichten gesetzlich nicht angehören dürfen, in den als Berufungsinstanz entscheidenden Ehrengerichtsh of berufen zu wollen; und man hat dies wohl bloss deshalb nicht ausgesprochen, weil man es eben als eine ganz selbstverständliche und unzweifelhafte Consequenz des $\S 2$ ansah. Es würde gewiss nicht schwierig sein, über die Meinung der Regierung in diesem Punkte noch bestimmte Aeusserungen nöthigenfalls zu extrahiren; vermuthlich werden schon die bei Einbringung des Gesetzentwurfes zu erwartenden Motive ganz unzweideutige, jedes Missverständniss ausschliessende Angaben in dieser Beziehung enthalten.

Fallen nun diese immer in den Vordergrund geschobenen Befürchtungen weg, durch eine wesentlich aus Beamten zusammengesetzte, von der Regierung künstlich geschaffene Mehrheit des Ehrengerichtshofes majorisirt zu werden, so lassen sich wohl die übrigen noch ausstehenden Differenzpunkte mit viel grösserer Gemüthsruhe erledigen. Es handelt sich dabei zunächst 
um die Heranziehung auch des ausserberuflichen Verhaltens der Aerzte ( $\$ 16$ des ersten Entwurfes). Die ältere Fassung; "in Ausübung des Berufs sowie ausserhalb desselben", ist in dem neuen Entwurfe umgangen, und es heisst hier nun ganz allgemein, dass „ein Arzt, welcher die Pflichten seines Berufes verletzt oder sich durch sein Verhalten der Achtung und des Vertrauens unw ürdig zeigt, welche der ärztliche Beruf erfordert", die ehrengerichtliche Bestrafung verwirkt liabe. Auch diese etwas dehnbare Fassung ist ja vielleicht noch verbesserungsfälig; indessen vom Standpunkte derer, die die ganze Einrichtung gerade hauptsächlich fordern, um unlauteren Elementen des Aerztestandes zu Leibe zu gehen, sollte man doch einigen Werth darauf legen, die dafür bestimmte Waffe nicht von vornherein allzusehr abzustumpfen und ihre zweckdienliche Anwendung unmöglich zu machen. Es ist doch im Grunde ziemlich inconsequent und nur aus einer den Aerzten nun einmal unvertilgbar anhaftenden, in diesem Falle zu weit getriebenen Empfindsamkeit, die sich gern den Deckmantel der „Humanität" ïberhängt, begreifbar, dass wir fortwährend nacl Waffen rufen und, sobald uns diese Waffen gewährt werden, eine Himmelangst empfinden, sie könnten auch einmal gegen uns selbst gekehrt und zur Abschlachtung unschuldiger Opfer missbräuchlich benutzt werden. Ja, wenn wir $\mathrm{zu}$ uns selbst und zu den von uns gewählten und eingesetzten Vertretern so wenig Vertrauen haben, dann thäten wir allerdings besser, auf das Spielen mit gefährlichem Schiessgewehr, wie Ehrengerichte und dgl., ein- für allemal zu verzichten. Und wer weiss - vielleicht thäten wir wirklich besser daran! vielleicht ist der ärztliche Stand für solche Institutionen gegenwärtig noch nicht reif und muss erst durch weitere schmerzliche Erlebnisse und Erfahrungen am eigenen Leibe dafür "erzogen" werden! In dem dritten Differenzpunkte, der auch dem Vertreter der Anklage gewährten Möglichkeit zur Einlegung der Berufung an den Ehrengerichtshof $(\$ 39)$, hat die Regierung, wie vorauszusehen war, keine Concession gemacht und nach den nun einmal allgemein gültigen Regeln des Strafverfahrens, die stets beiden Parteien das gleiche Recht wahren, wohl auch nicht machen können. Dieser Punkt dïrfte jedoch einerseits gegenüber der veränderten (und eventuell noch weiter zu verändernden) Fassung des $\S 13$, andererseits gegenüber der inehr im Sinne der Aerzte veränderten Zusammensetzung des Ehrengerichtshofes $(\S 43)$ sehr an Bedeutung verlieren. Die Hauptsache ist doch, dass der Beschuldigte auch in der Berufungsinstanz nur von seinen näheren Berufsgenossen, nicht von einem überwiegend aus abhängigen Beamten bestehenden Collegium abgeurtheilt werden kann - was, ich wiederhole es, meiner Meinung nach durch die richtig verstandenen Bestimmungen des $\S 43$ jetzt vollkommen gewährleistet wird. - Bei dieser Sachlage muss man sich denn doch fragen, ob die ausserdem noch bestehenden unwesentlichen Differenzpunkte ausreichen, um, wie das von einzelnen Heissspornen sclion jetzt dringend gefordert wird, zu einer summarischen Ablehnung des Gesetzentwurfes in seiner nun vorliegenden Gestalt Anlass zu geben. Man muss doch nicht ganz vergessen, dass dieser Gesetzentwurf nicht aus der Initiative der Regierung liervorgegangen, noch weniger (wie es ein Theil der Presse darzustellen beliebt) von der Regierung den ahnungslosen Aerzten gewissermaassen aufoctroyirt worden ist sondern, dass viele Jahre hindurch aus Aerztekreisen und zumal von Seiten der berufenen Aerztevertretungen immer und immer wieder Anregungen, Wünsche, zuletzt stürmische Forderungen in dieser Richtung erhoben und kundgemacht wurden. Diesen Wünschen und Forderungen hat die Regierung im Interesse des ärztlichen Standes endlich nachgeben zu sollen geglaubt, sie hat ihren Gesetzentwurf vorgelegt und auch den auf seine partielle Abänderung hinzielenden Vorschlägen immerlin nicht unwesentliche Concessionen gemacht; sie wird aber dem ganzen Entwurfe, wenn nichts daraus werden sollte, vermuthlich keine Thräne nacliweinen, sondern sich bei dem alten Rechtssatze beruhigen: „Beneficia non obtruduntur". Sache der Aerzte aber wird es sein, sich bei den demnächst bevorstehenden Berathungen dieser Erwägung in letzter Stunde nicht zu verschliessen! A. E. 